\title{
Sub-surface Serial Block Face Scanning Electron Microscopy
}

\author{
Q. He ${ }^{1}$, M.A. Aronova ${ }^{1}$, D.C. Joy ${ }^{2,3}$, G. Zhang ${ }^{1}$, R.D. Leapman ${ }^{1}$
}

${ }^{1}$ National Institute of Biomedical Imaging and Bioengineering, NIH, Bethesda, MD 20892;

${ }^{2}$ Department of Materials Science and Engineering, University of Tennessee, Knoxville, TN 37996;

${ }^{3}$ Center for Nanophase Materials Sciences, Oak Ridge National Laboratory, Oak Ridge, TN 37831

Serial block face (SBF) imaging in the scanning electron microscope (SEM) provides nanoscale 3D ultrastructure of biological samples up to several hundred micrometers in size. In SBF-SEM, an ultramicrotome built into the SEM specimen stage successively removes thin sections from a plasticembedded, heavy metal-stained specimen. After each cut, the freshly exposed block face is imaged at a low incident electron energy using the backscattered electron signal, which is sensitive to heavy atoms in the sample. Although the $\mathrm{x}-\mathrm{y}$ resolution in the plane of the block face is approximately 5 $\mathrm{nm}$, the resolution along the z-axis in SBF-SEM is limited by the minimum slice thickness of around $25 \mathrm{~nm}$. We have explored the feasibility of improving the z-resolution in SBF-SEM by recording images at more than one primary beam energy, thus sampling different depths below the block surface $[1,2]$.

An illustration of the relationship between primary beam energy and penetration depth is shown in Fig. 1A. We used Monte Carlo simulations of SEM images from an epoxy block containing 5-nm diameter carbon spheres stained with $14 \%$ lead positioned at different depths, as a model for small biological structures within cells (Fig. 1B) [3]. A linear relationship was found between the depth of the spheres and the ratio of backscattered images recorded at primary beam energies of $1.4 \mathrm{keV}$ and $6.8 \mathrm{keV}$, which allowed us to determine 3D structure within a $25-\mathrm{nm}$ surface layer with a depth resolution of around $5 \mathrm{~nm}$. The model obtained from the simulation was tested experimentally on a specimen consisting of 5-nm gold spheres embedded in a multilayer carbon film using a Zeiss Sigma-VP SEM equipped with a Gatan 3View SBF system. BSE images recorded at primary beam energies of $2.4 \mathrm{keV}$ and $6.8 \mathrm{keV}$ are shown in Fig. 2A and 2B. The computed 3D model is visualized in Figs. 2C and 2D, along with $5 \mathrm{~nm}$ calculated slices at depths of $0 \mathrm{~nm}, 5 \mathrm{~nm}, 10 \mathrm{~nm}, 15 \mathrm{~nm}, 20 \mathrm{~nm}$, and $25 \mathrm{~nm}$ (Figs. D-I). Experiments were also performed on embedded blocks of stained biological tissues to demonstrate that 3D structure could be determined from 25-nm thick sub-surface layer as shown in Fig. 3, where the heavy-atom stain appears bright in the BSE images.

Although damage of the block under electron irradiation limits the signal to noise ratio, sub-surface serial block face SEM using multiple primary beam energies provides the possibility of obtaining nearly isotropic 3D spatial resolution [4].

\section{References}

[1] P Hennig and W Denk, Journal of Applied Physics 102 (2007), p.123101-1.

[2] F Boughorbel et al, FEI Compnay, US Patent (\#US8,232,523 B2) 2011.

[3] H Demers et al, Scanning 33 (2011), p.135.

[4] This research was supported by the intramural program of the National Institute of Biomedical Imaging and Bioengineering, NIH. 


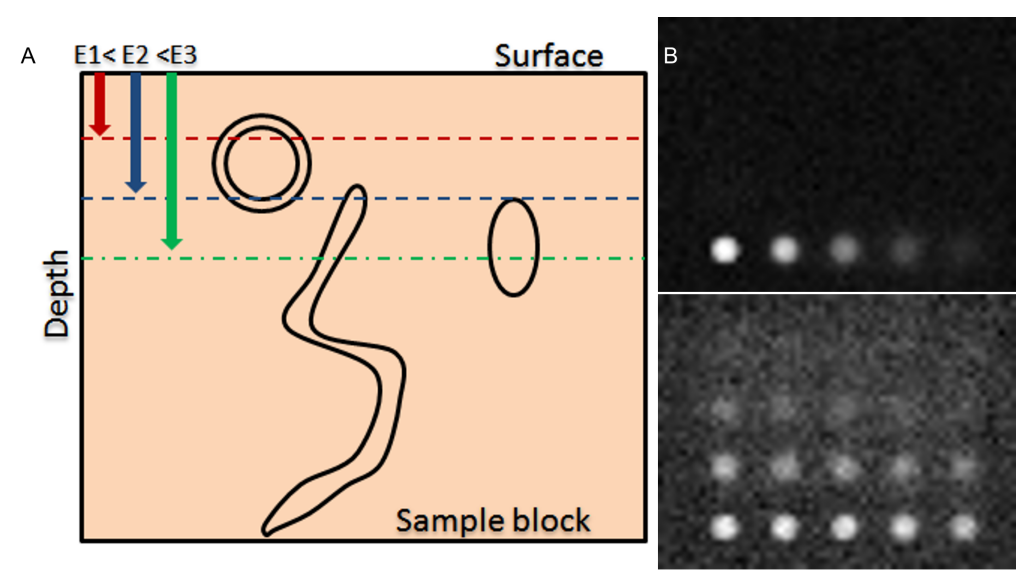

Fig. 1. (A) Relationship between primary beam energy and penetration depth in a resin-embedded block of stained cell: higher beam energy $E_{3}$ leads to deeper penetration than lower energies of $E_{2}$ and $\mathrm{E}_{1}$. (B) Monte Carlo simulation of stained spheres containing $14 \% \mathrm{~Pb}$ in an epoxy resin matrix; upper image, $1.4 \mathrm{keV}$ beam energy; lower image, $6.8 \mathrm{keV}$ beam energy.
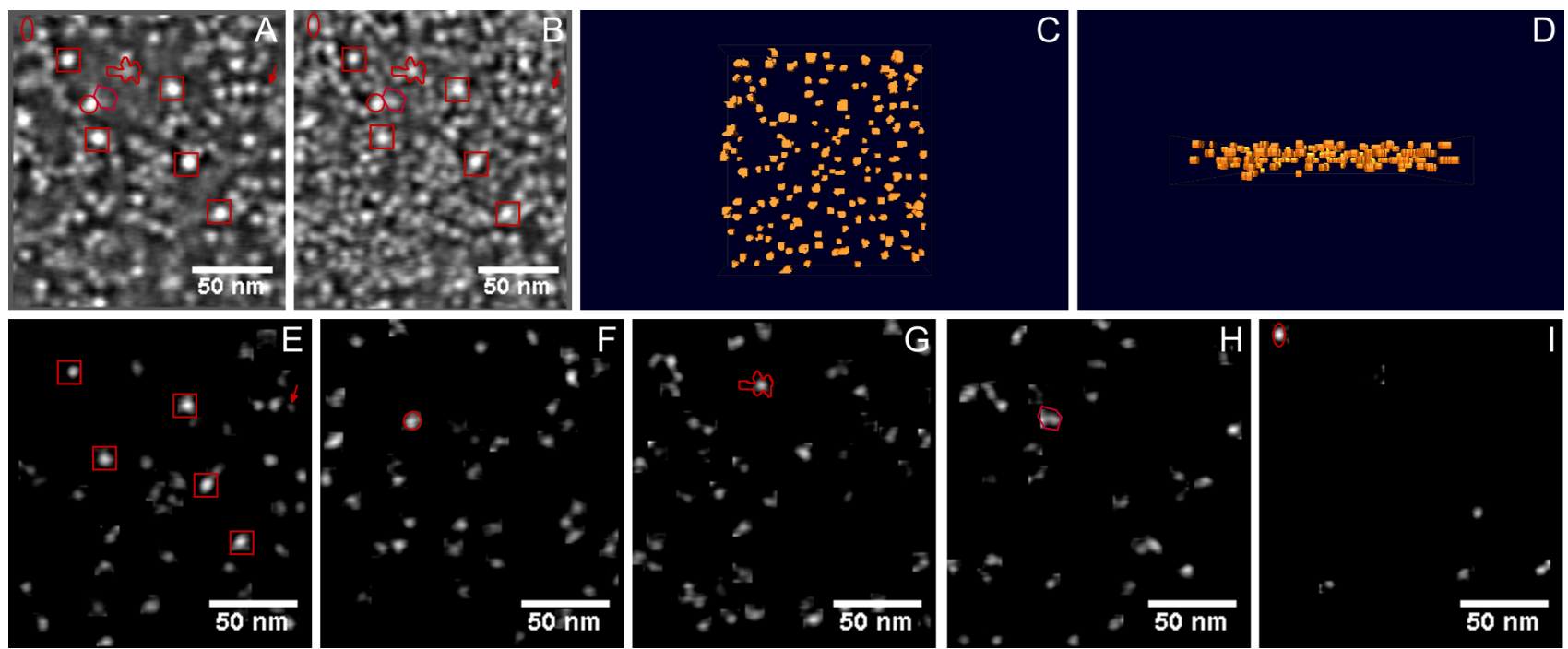

Fig. 2. BSE images of specimen consisting of 5-nm diameter Au nanoparticles embedded in carbon film, acquired at primary beam energies of (A) $2.4 \mathrm{keV}$ and (B) $6.8 \mathrm{keV}$; (C) calculated 3D model in top view, and (D) in side view. Panels E-I are computed 5-nm thick slices at depths of (E) 0-5 nm, (F) 5-10 nm, (G) 10-15 nm, (H) 15-20 nm and (I) 20-25 nm.
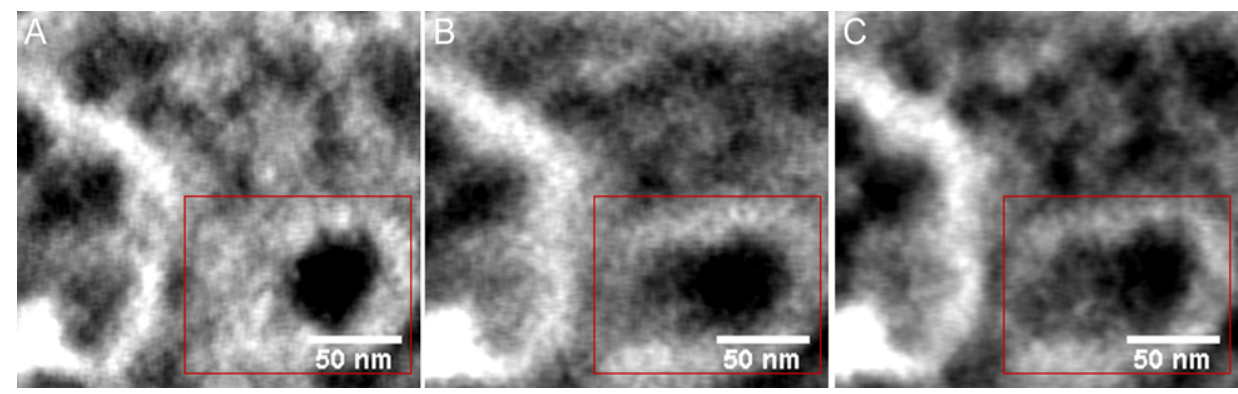

Fig. 3. BSE images of sub-region of beta cell in pancreatic islet of Langerhans acquired at different primary energies of (A) $1.4 \mathrm{keV}$, (B) $2.4 \mathrm{keV}$, and (C) $3.4 \mathrm{keV}$. Differences in appearance of membrane structure (in box) are due to variations in stain as a function of depth. 